\title{
Bi-directional Route Optimization in Mobile IP over Wireless LAN
}

\author{
Chun-Hsin Wu, Ann-Tzung Cheng, Shao-Ting Lee, Jan-Ming Ho and D. T. Lee \\ Institute of Information Science \\ Academia Sinica \\ Taipei, Taiwan \\ \{wuch, jahorng, kingking, hoho, dtlee\}@iis.sinica.edu.tw
}

\begin{abstract}
In this paper we propose to support symmetric bidirectional route optimization in mobile IPv4 considering ingress-filtering routers. We present subnet-based direct tunneling techniques to improve the routing efficiency for mobile IP and a binding optimization technique to reduce the handoff latency for mobile nodes. An enhanced correspondent agent was introduced to collaborate with the home agent and the foreign agent to support these techniques. Utilizing a tunneling cache, datagrams sent from a mobile node can be directly tunneled to a correspondent agent, including Binding Update messages. The proposed approach can maximize the use of a bi-directional tunnel while maintaining transparency to existing hosts. Based on our deployment and simulation experiment, the enhanced mobile IP protocol is suitable for wireless LANs that provide broadband multimedia and location-based services.
\end{abstract}

Keywords—mobile IP; route optimization; triangle routing; ingress-filtering routers; reverse tunneling

\section{INTRODUCTION}

One of the most critical challenges to providing mobility at the IP layer is to route packets efficiently and securely. In the base mobile IP protocol [1], all packets sent to a mobile node while away from home are intercepted by its home agent and tunneled to the mobile node using IP encapsulation within IP [2][3][4]. This allows transparent routing of IP datagrams to the mobile node, but it raises a serious efficiency issue known as the triangle routing problem where packets have to take a long detour.

To reduce the routing overhead in mobile IP, the route optimization approach was then proposed to directly tunnel packets from a sending node to a foreign agent where the recipient mobile node is visiting [5][6]. Following this approach, a route-optimized correspondent node may obtain the binding of home address to corresponding care-of address of mobile nodes from the binding update messages sent by the home agent, and maintain a binding cache to optimize its own communication with these mobile nodes. Although this would improve the performance of packet routing in mobile IP, it requires that the correspondent node be enhanced to support mobile IP.
To retain transparency to the correspondent node, an agent-based route optimization approach was proposed to move the tasks of maintaining binding caches and encapsulating messages performed by individual correspondent nodes to the correspondent agent [7]. In addition, a tunnel set up by one correspondent agent could serve multiple correspondent nodes simultaneously. This would reduce the total number of control messages needed to set up individual tunnels for all correspondent nodes nearby the correspondent agent.

Although the above route optimization techniques can improve the routing efficiency in mobile IP to a certain extent, they focus on the routing direction from the correspondent node to the mobile node and they may not work well in most networks that follow strict and secure routing policies. For example, to defeat denial of service attacks which employ IP source address spoofing, many routers begin to adopt filtering strategy and will drop a packet whose source address is not topologically correct [8]. These network ingress-filtering routers disallow a mobile node to send packets directly from the visited foreign network to correspondent nodes using its home address as the source address.

To counteract this ingress filtering measure, the reverse tunneling scheme was proposed to establish a topologically correct reverse tunnel from the care-of-address of a mobile node to the home agent [9]. The packet sent from a mobile node is reverse tunneled to its home agent and then forwarded to the destined correspondent node after de-tunneling. This scheme results in the triangle routing problem in the reverse direction and makes the current route optimization techniques become unidirectional or asymmetric.

Supporting direct routing in correspondent nodes and direct tunneling in mobile nodes, bi-directional route optimization is now built as a fundamental part in the work-inprogress mobile Ipv6 [10]. In the proposal, a mobile node uses its case-of address as the source address in the IP header of packets it sends. It may also directly send new binding update messages to certain correspondent nodes to reduce handoff latency. Besides, most packets sent to a mobile node while away from home are sent using an IPv6 routing header 
rather than IP encapsulation in route optimization of mobile IPv4.

In this paper we examine the direct tunneling approach to solving the triangle routing problem in mobile IPv4with ingress filtering. In addition to adapting the techniques in the mobile IPv6 draft to mobile IPv4, we propose new subnetbased tunneling and routing optimization techniques to optimize packet routing further. The enhanced mobile IP protocol supports symmetric bi-directional route optimization to improve the performance of both packet transmission and mobile-node handoff.

This paper is organized as follows. In Section II, we introduce an application of mobile IP over wireless LAN in a regional guidance system and make a few observations about such kind of systems. The proposed techniques will improve the mobile IP performance based on these observations. We present the architectural design and operations of the proposed bi-directional route optimization in Section III and Section IV, respectively. In Section V, we present our implementation experience of developing a campus guidance system with mobile IP over wireless local area networks (WLAN) that support broadband multimedia and location-based services. Section VI concludes the paper.

\section{MobILE IP OVER WIRELESS LAN IN A REGIONAL GUIDANCE SYSTEM}

With the rapid progress in the development of wireless technology, recent advancements in wireless local area networks have shown their promises to high-rate wireless data transmission [11][12]. Complementing the operator's existing GSM and GPRS services [13], wireless LAN technology provides a means for cost-effective broadband Internet access. Not only are WLANs installed in indoor offices, but many of them are also deployed in public spaces and campuses already [14][15].

At present, WLAN like IEEE $802.11 \mathrm{~b}$ can transmit data up to 100 meters [16]. As the transmission distance increases, WLAN may also provide an economic solution for outdoor wireless data transmission, especially in regional areas such as campuses or parks. Operating in the unlicensed industrial, scientific and medical (ISM) bands, a wireless network with WLAN can be built within a university or organization and the existing wired backbone can be used to provide broadband access for the whole campus. In addition to providing Internet access, we can also develop a campus guidance system that provides multimedia and navigation services by supporting IP mobility with WLAN. Aided with a positioning system [17], a visitor can then be navigated in a campus with a hand-held device or on-board unit and toured by location-based multimedia services through the WLAN [18].

A campus guidance system with mobile IP over wireless LAN can work as an experimental testbed for wireless broadband. A mobile IP architecture for the system can be

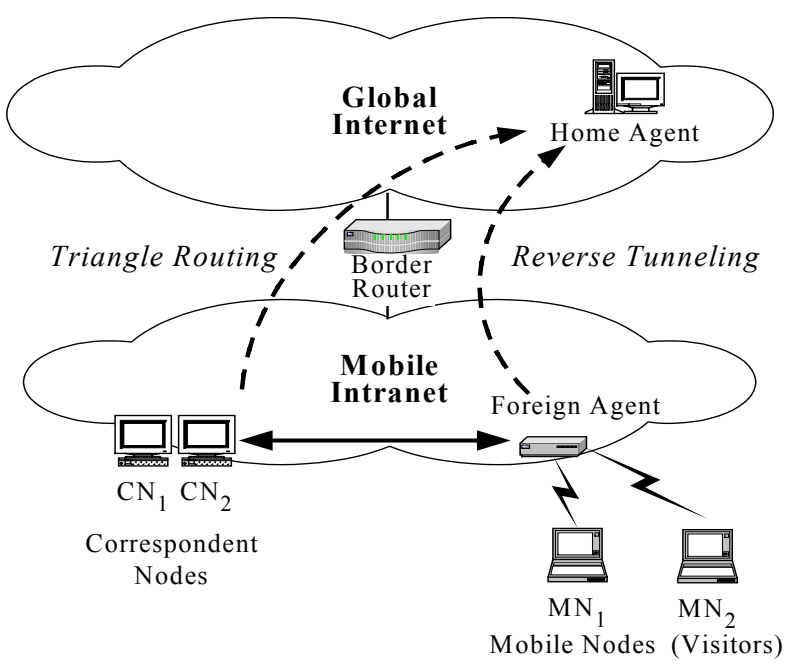

Figure 1. Routing and tunneling packets in mobile IP over wireless LAN.

depicted as shown in Fig. 1. In the system a foreign agent is located at a nearby access point. A department may run Internet servers as correspondent nodes to provide locationbased multimedia contents. A visitor can connect to the mobile campus Intranet through a foreign agent with a mobile node and he can also access the outside global Internet as usual. For such systems, we can have a few observations:

- In the Internet, most applications are developed in the client-server model where the interaction of service is started by client's request. This implies that the connection between a mobile node and a correspondent node is most likely initiated by the mobile node.

- Most content providers build up groups of servers to provide contents in the Web. A user requesting contents from one provider in the same session may connect to more than one server of the provider. It means that a mobile node may access more than one correspondent node in the same subnet during one session.

- Specifically in a regional guidance system, visitors are usually from outside the region and probably interested in navigation and location-based services provided by servers inside. This suggests that in the system, home agents of the mobile nodes are usually outside the mobile Intranet and many correspondent nodes of interest to the mobile nodes are inside. So delivering packets using triangle routing and reverse tunneling for such a system will not only cause long access latency for mobile nodes, but also generate unnecessary traffic to the border router and home agents.

- Neighboring visitors arriving in the same cell or access point might desire similar local contents, so it would not be unusual that two mobile nodes supported by the same foreign agent retrieve similar location-based contents from the same or surrounding correspondent nodes. 
- Providing mobile Internet access with WLAN, handoff may occur more frequently than existing cellular networks due to shorter transmission range. However, because the speed limit in a campus is usually low, slower walking or driving speed may prevent handoff from happening too fast to proceed.

Most current mobile IP solutions optimize the routes from correspondent nodes to mobile nodes individually. These observations indicate that there is room for improvement to build a more optimized guidance system with mobile IP over WLAN. .

\section{ARCHITECTURAL DESIGN}

This section presents the overall design of our bidirectional route optimization. Not only is the proposed scheme aimed to address the issues of triangle routing and ingress filtering in mobile IPv4, it is also aimed to enhance route optimization based on the observations described in the previous section. In addition, the proposed approach needs to function well with traditional Internet hosts just like the base mobile IPv4 that allows any existing Internet hosts to communicate with a mobile node as usual.

As shown in Fig. 2, our design introduces a correspondent agent to retain the transparency to correspondent nodes and to serve multiple correspondent nodes of the same subnet for route optimization. The correspondent agent maintains the binding cache proposed by [5] and intercepts all packets sent to and from the correspondent nodes.

Symmetrically, a foreign agent, at the other end of the optimized route or tunnel, maintains a tunneling cache for bidirectional route optimization. An entry of the tunneling cache indicates that a correspondent node or correspondent network supports route optimization and direct tunneling, so a foreign agent can directly tunnel a packet received from a mobile node to the correspondent node that matches a tunneling cache entry. In the original route optimization, any node may maintain a binding cache to optimize its own communication with mobile nodes [5]. Similarly, in the bi-directional route optimization, any node may maintain a tunneling cache to optimize its own communication with the correspondent nodes.

When a foreign agent receives a packet tunneled from a correspondent agent or a correspondent node, it will assume the sender support direct tunneling and then create or update a tunneling cache entry for the sender. So there are no additional messages required to create or update a tunneling cache entry. This is different from the maintenance of binding caches where a correspondent node creates or updates a binding cache entry when it has received a binding update message to change a mobile node's mobility binding.

Note that all the route-optimized packets are tunneled either by a correspondent agent or by a foreign agent. Before a tunnel is setup, neither the foreign node nor the correspondent

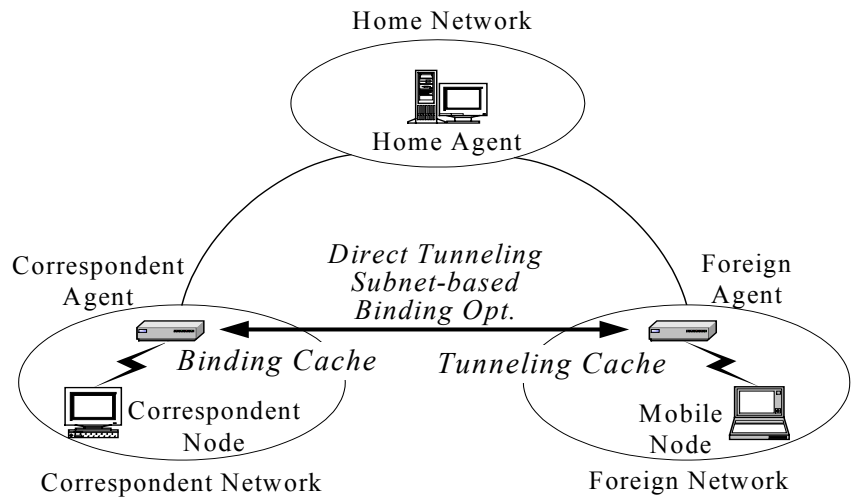

Figure 2. Architecture of bi-directional route optimization.

node knows whether the other endpoint supports direct tunneling. So a home agent or a foreign agent must be able to distinguish a traditional correspondent node from an enhanced correspondent agent, and the enhanced correspondent agent must also be able to distinguish a mobile node from a usual stationary node. All of these agents can be implemented as an extension to ordinary IPv4 routers that intrinsically intercept passing packets.

\section{OPERATIONS OF BI-DIRECTIONAL ROUTE OPTIMIZATION}

\section{A. Bi-directional Direct Tunneling}

Since a correspondent agent stays with a router, it will intercept all packets involving the correspondent nodes. When a correspondent agent intercepts a packet sent from a correspondent node and it does not have a binding cache entry for the receiver of the packet, the correspondent agent will forward the packet as an ordinary IP datagram. As specified in the original route optimization protocol [5], this normal packet may trigger the home agent to send a binding update message to the sending correspondent node. While intercepting this binding update message, the correspondent agent will create a new binding cache entry. It might also start to establish a mobility security association with the home agent.

When a correspondent agent intercepts a packet sent from a correspondent node and it has a binding cache entry for the destination mobile node, it will encapsulate and tunnel the packet to the responsible foreign agent. While receiving this tunneled packet, the foreign agentwill de-tunnel and forward the packet to the destination mobile node. Meanwhile, the foreign agent will also recognize that the correspondent network with the sending correspondent node supports route optimization, so it will create or update its tunneling cache entry accordingly.

Reversely, when a foreign agent receives a packet sent from a visiting mobile node to a correspondent node that matches a tunneling cache entry, it will encapsulate and tunnel the packet to the destination correspondent node. If there is no tunneling cache entry suitable for the destination of a packet, 
it will reverse tunnel the packet to the mobile node's home agent as usual [9]. While the responsible correspondent agent intercepts this packet tunneled from the foreign agent, it detunnels the packet and forwards it to the destination correspondent node.

As described, the proposed optimization technique will solve the triangle routing problem for both directions in mobile IP, even with routers running ingress-filtering. It can reduce the transmission latency for packets, and the network traffic for the home agent and the border router.

\section{B. Subnet-based Route Optimization}

To support subnet-based tunneling, a correspondent agent will put the subnet mask of the correspondent network in an encapsulated packet from a correspondent node to a mobile node, and the foreign agent receiving this packet will keep the subnet information in its tunneling cache. Hereafter a foreign agent uses the subnet information to decide whether a correspondent node is behind a correspondent agent that supports direct tunneling. When a mobile node wants to send a packet to any correspondent node in the route-optimized correspondent network, the foreign agent can directly tunnel the packet to the destination through the responsible correspondent agent without passing through the home agent. To prevent a packet sent from a mobile node to a correspondent node from being hijacked, a foreign agent should use the destination address of the packet as the outer destination address of the encapsulated packet.

In addition to reducing the size of binding and tunneling caches anywhere, this subnet-based route optimization can also reduce the number of control messages for establishing and maintaining the tunnels to individual nodes. Another major advantage is to maximize the possibility of reusing the optimized route. Reconsider Fig. 1 and Fig. 2. Assume the route between $\mathrm{CN}_{1}$ and $\mathrm{MN}_{1}$ was established such that there is a binding cache entry for the mobile node in the correspondent agent and a tunneling cache entry for the correspondent network in the foreign agent. There are three more ways to reuse the tunnel between $\mathrm{CN}_{1}$ and $\mathrm{MN}_{1}$ without additional establishment or maintenance messages:

1) Sending packets from $\mathrm{MN}_{1}$ to $C N_{2}$

2) Sending packets from $\mathrm{CN}_{2}$ to $M N_{1}$

3) Sending packets from $\mathrm{MN}_{2}$ to $\mathrm{CN}_{1}$ or $\mathrm{CN}_{2}$

Case 1 and case 3 utilize the tunneling cache entry and case 2 utilizes the binding cache entry. Note that because the mobility binding of a mobile node requires an explicit binding update message, the route between $\mathrm{CN}_{1}$ and $\mathrm{MN}_{1}$ does not deduce the reuse of the tunnel to send packets from $\mathrm{CN}_{1}$ or $\mathrm{CN}_{2}$ to $\mathrm{MN}_{2}$.

Different from conventional route optimization schemes that optimize the route to individual nodes, the proposed bidirectional route optimization scheme can optimize the route to subnets. While establishing the mobility security association or setting up a tunnel, the control message and the tunneled packet between different agents may also be associated with additional subnet information. Applying this technique, no extra messages are required to support subnetbased route optimization.

\section{Binding Optimization}

In the current route optimization proposal, when a mobile node moves and an IP-handoff occurs, it relies on the home agent, which keeps a binding update list, to send Binding Update messages to all of its correspondent nodes [5]. Since the foreign agent in our proposal can transmit packets directly to mobility-aware correspondent agents, the foreign agent can also send Binding Update messages directly by itself to the correspondent agents when the mobile node moves away. This binding optimization technique can further reduce the handoff delay especially for those active tunnels without triangularly transmitting the binding update information through the home agent. However, to support backward-compatibility with the base mobile IP and route optimization protocols, the enhanced foreign agent still needs to send a Binding Warning message to the home agent to notify the home agent and the ordinary correspondent nodes that the mobility binding is updated.

\section{IMPLEMENTATION EXPERIENCE}

In order to explore the issues of mobile computing and wireless networking, we are developing a regional guidance system with $802.11 \mathrm{~b}$ WLAN covering the major roads in the campus of Academia Sinica. At present ten $802.11 \mathrm{~b}$ access points as the base stations are scheduled for the testbed and three of them along a road have been installed. Each access point equipped with outdoor antenna was placed near the window of the building and connected to the existing IP backbone in Academia Sinica. The transmission quality is still very well even if two access points are 100-meter apart.

We have implemented the proposed bi-directional route optimization techniques in our AutoPC prototype [18]. The AutoPC equipped in the car is a part of our campus guidance system that provides broadband mobile Internet access over the $802.11 \mathrm{~b}$ wireless LAN. We have also developed in-vehicle navigation and location-based wireless multimedia streaming services for the AutoPC. When a visitor arrives at the main gate, he will be navigated to his destination and given brief introduction to the building near him.

From our preliminary results, the handoff latency can be reduced below one second and we can continuously play multimedia streams smoothly with the support of stream buffering. The packet transmission time and the traffic in the border router and the home agent can also be reduced dramatically through direct tunneling. Further simulation is under development. Testing our system in the environment of multiple $802.11 \mathrm{~b}$ cells, the experimental results showed that an in-vehicle AutoPC could run video streaming application 
smoothly on the road even when the car travels at a speed up to $50 \mathrm{~km} / \mathrm{hr}$.

\section{CONCLUSION}

Applying the proposed subnet-based direct tunneling and binding optimization techniques, symmetric bi-directional route optimization can be supported for mobile IP even if the ingress-filtering routers in the network drop topologically incorrect packets. The presented solution not only improves the efficiency of packet routing, but also reduces the handoff latency for mobile nodes. Our experience has demonstrated the feasibility of mobile IP over wireless LANs.

\section{REFERENCES}

[1] C. Perkins, "IP Mobility Support for IPv4," RFC 3220, January 2002.

[2] S. Hanks, T. Li, D. Farinacci and P. Traine, "Generic Routing Encapsulation (GRE)," RFC 1701, October 1994.

[3] C. Perkins, "IP Encapsulation within IP," RFC 2003, October 1996.

[4] C. Perkins, "Minimal Encapsulation within IP," RFC 2004, October 1999.

[5] D. Johnson and C. Perkins, "Route Optimization in Mobile IP," Internet Draft draft-ietf-mobileip-optim-11.txt (work in progress), September 2001.

[6] D. Johnson, "Scalable and Robust Internetwork Routing for Mobile Hosts," in Proceedings of IEEE $14^{\text {th }}$ International Conference on Distributed Computing Systems, June 1994.

[7] R. Vadali, J. Li, Y. Wu and G. Cao, "Agent-Based Route Optimization for Mobile IP," in Proceedings of IEEE $54^{\text {th }}$ Vehicular Technology Conference, October 2001.
[8] P. Ferguson and D. Senie, "Network Ingress Filtering: Defeating Denial of Service Attacks which Employ IP Source Address Spoofing," RFC 2267, 1998.

[9] G. Montenegro, "Reverse Tunneling for Mobile IP, revised," RFC 2344, 2001.

[10] D. Johnson, C. Perkins and J. Arkko, "Mobility Support in IPv6," Internet Draft draft-ietf-mobileip-ipv6-17.txt (work in progress), May 2002.

[11] R. van Nee, G. Awater, M. Morikura, H. Takanashi, M. Webster and K. Halford, "New High-Rate Wireless LAN Standards," IEEE Communications Magazine, vol. 37, no. 12, pp. 82-88, December 1999.

[12] R. Jordan and C. Abdallah, "Wireless Communications and Networking: An Overview," IEEE Antenna's and Propagation Magazine, vol. 44, no. 1, pp. 185-193, February 2002.

[13] J. De Vriendt, P. Laine, C. Lerouge and X. Xu, "Mobile Network Evolution: A Revolution on the Move," IEEE Communications Magazine, vol. 40, no. 4, pp. 104-111, April 2002.

[14] A. Hills, "Large-Scale Wireless LAN Design," IEEE Communications Magazine, vol. 39, no. 11, pp. 98-104, November 2001.

[15] J. Ala-Laurila, J. Mikkonen and J. Rinnemaa, "Wireless LAN Access Network Architecture for Mobile Operators," IEEE Communications Magazine, vol. 39, no. 11, pp. 82-89, November 2001.

[16] "IEEE 802.11 Local and Metropolitan Area Networks: Wireless LAN Medium Access Control (MAC) and Physical (PHY) Specification," ISO/IEC 8802-11, 1999.

[17] C. Drane and C. Rizos, Positioning Systems in Intelligent Transportation Systems. Boston; Artech House, Inc., 1997.

[18] C.H. Wu, A.T. Cheng, S.T. Lee and J.M. Ho, "An AutoPC for Supporting In-Vehicle Navigation and Location-Based Multimedia Services," in Proceedings of IEEE Position Location and Navigation Symposium, April 2002. 